\title{
Quaderni
}

QUADERNI Communication, technologies, pouvoir

67 | Automne 2008

Jeu vidéo et discours

\section{Soigner des jeux vidéo / soigner par les jeux vidéo}

Regards croisés sur un révélateur de mal-être

Healing from / with videogames

\section{Thomas Gaon et Michel Stora}

\section{(2) OpenEdition}

Journals

Édition électronique

URL : http://journals.openedition.org/quaderni/192

DOI : 10.4000/quaderni. 192

ISSN : 2105-2956

Éditeur

Les éditions de la Maison des sciences de l'Homme

\section{Édition imprimée}

Date de publication : 5 octobre 2008

Pagination : 33-42

\section{Référence électronique}

Thomas Gaon et Michel Stora, «Soigner des jeux vidéo / soigner par les jeux vidéo », Quaderni [En

ligne], 67 | Automne 2008, mis en ligne le 05 janvier 2012, consulté le 30 avril 2019. URL : http://

journals.openedition.org/quaderni/192; DOI : 10.4000/quaderni.192 


\section{$D$ ossier}

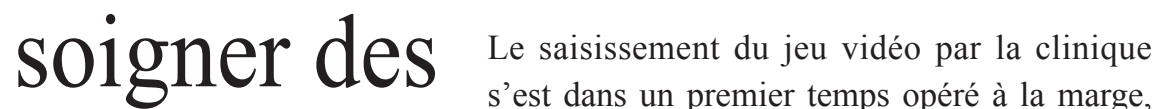
jeux vidéo / notamment autour des questions d'addiction. Les premières consultations sont venues d'une demande sociale, mais l'offre de service n'étant soigner par les jeux vidéo regards croisés sur un révélateur de mal être

Thomas

Gaon

psychologue clinicien, centre d'addictologie le littoral

\section{Michel Stora} psychanalyste

membres fondateurs de l'Observatoire des Mondes Numériques en Sciences Humaines (OMNSH) à l'époque pas constituée, les premiers patients ont été acceptés dans les services de toxicomanie. Car le meilleur moyen de déterminer si un joueur peut souffrir d'une addiction est d'analyser si la pratique excessive pouvait être une pathologique, puis quelle serait cette pathologie, Thomas Gaon revient sur la carrière de la notion d'addiction, dans une généalogie critique du concept. Pour autant, les jeux de par leur nature d'objet transitionnel ${ }^{1}$ sont des supports de médiation privilégiés. Dans une approche psychanalytique développée par Michael Stora, il devient possible d'utiliser les jeux vidéo comme outil capable de matérialiser les angoisses et troubles des plus jeunes, parfois incapables de les formuler autrement que par les postures qu'ils engagent vis-àvis des jeux vidéo. Dans cette approche croisée sur l'usage médical des jeux vidéo, il apparaît qu'ils sont ainsi davantage un révélateur de mal être, que le réel déclencheur de pathologies.

\section{Critique de la notion d'addiction aux jeux} vidéo

La notion d'addiction aux jeux vidéo se construit à partir des éléments suivants : une réalité clinique, la méconnaissance de l'objet jeu vidéo, la mutation de la psychiatrie moderne, une ambiguité terminologique et la gestion thérapeutique captée par l'addictologie.

Au tournant du millénaire, l'arrivée des jeux en ligne modifie les pratiques ludiques notamment 
en ôtant la contrainte locale de partenaires physiques et en renforçant l'interdépendance des joueurs. Les propriétés intrinsèques des jeux de rôle en ligne (MMORPG), dont l'absence de limite interne est caractéristique, permettent des usages problématiques ${ }^{2}$ qui émergent dès 1999 sur Everquest ${ }^{3}$. Avec la démocratisation du MMORPG et de sa pratique, le nombre d'usages excessifs augmente et leur proportion varie entre 5 et $12 \%$ des joueurs de MMORPG selon les estimations actuelles ${ }^{4}$.

L'objet en question n'est donc pas le jeu vidéo dans sa globalité mais uniquement un type de jeu vidéo en ligne dont les propriétés et la présence justifie en partie la possibilité d'un usage excessif.

S'il existe indéniablement une réalité clinique d'usages problématiques du MMORPG, sa qualification en terme d'addiction est extrêmement problématique car ce concept psychiatrique - tel qu'il est évoqué actuellement - relève de la visée classificatoire de la psychiatrie moderne et possède un imaginaire florissant issu de la représentation de la drogue qui oblitère sa compréhension.

Sous les influences de la psychopharmacologie ${ }^{5}$ et de la neurobiologie d'une part et des mutations politiques et économiques mondiales d'autre part, la psychiatrie internationale moderne a dû s'inscrire dans une scientificité basée sur l'épidémiologie et trouver un large consensus entre ses différents courants de pensée ${ }^{6}$. L'approche descriptive et classificatoire des troubles psychiatriques dont le Manuel Diagnostic et Statistique des troubles mentaux (DSM) rend compte, scelle le mode de « la juxtaposition des pratiques et de la superposition des théories $\rangle^{7} \mathrm{du}$ milieu psychiatrique. Dès lors, le fronton commun et visible de la psychiatrie se réduit dans sa communication extérieure à une liste de troubles mentaux définis par leurs signes, leur forme, et non plus par leur fond, leur nature précise et leurs causes - sujettes aux polémiques internes.

À partir de cette normalisation sémiologique ${ }^{8}$, l'intérêt heuristique concernant l'addiction réside dans la mise en lumière d'un ensemble de mécanismes psychiques et neurobiologiques qu'un humain éprouve de façon anormale lorsqu'il s'adonne à un plaisir ou échappe à une tension interne par l'entremise d'un comportement tel que l'usage de produit psychoactifs ou le jeu d'argent et de hasard, la sexualité, etc. D'un autre côté, la perspective descriptive délaisse le sujet, son idiosyncrasie, son histoire et son environnement. Pris uniquement sous l'angle des symptômes, de ces comportements qui troublent, la psychiatrie moderne ne possède plus de vision globale du sujet conçu désormais comme un porteur de troubles éventuellement additionnels dont les thérapeutiques spécialisées doivent le débarrasser ou en réduire l'impact. Cette conception galénique s'oppose à la conception hippocratique d'une maladie mentale comme faisant partie du sujet ${ }^{9}$. Les causes de la pathologie, l'histoire du sujet et surtout le sens que la maladie peut prendre n'ont désormais plus d'intérêt pour la classification des troubles adossée à la visée thérapeutique chimiothérapique ou cognitivo-comportementale principalement utilisée aux USA. La réification descriptive des troubles psychiatriques se substitue à leur compréhension et à leur signification subjective et sociale. 
En France, l'ambiguïté du terme d'addiction provient de ses quatre origines différentes. «Addiction » est un terme originairement psychiatrique et nord-américain qui est l'équivalent de la toxicomanie française (drug-addiction). Mais devant l'extension du terme sous la vague hippie ${ }^{10}$ et le détournement récent opéré par la publicité et les médias qui ont popularisé et vulgarisé le terme «addiction » vers des équivalents de accroc, mordu ou passionné ${ }^{11}$, la psychiatrie mondiale (leur) a abandonné le terme d' " addiction » pour « dependance », terme plus médical sous-tendant la présence de produits psycho-actifs. Une autre complication sémantique survient avec l'introduction des conduites de dépendances sans produit $^{12}$ que le psychiatre A. Goodman a tenté de réunir avec la toxicomanie et l'alcoolisme en une unique entité sous le terme générique de trouble addictif ou Addiction. Si la proposition de A. Goodman n'a pas été intégrée dans les classifications internationales, elle aura néanmoins remporté un succès auprès des addictologues qui trouvent ici leur bannière commune.

Le mot addiction fusionne donc l'ancienne toxicomanie, les addictions modernes avec et sans produits et un mode de consommation non pathologique soutenu par le commerce et les médias. Cette polysémie prête le flanc à la confusion suivante : «L'accroc, à quoi que ce soit, est (semblable à) un drogué ».

Il existe enfin une dernière acception d'addiction issue de la psychanalyse française ${ }^{13}$ et basée sur son étymologie. L'addiction est considérée ici comme une fonction protectrice du sujet par le recours répété au comportement. Elle serait liée à un défaut de symbolisation due à une faille précoce de l'environnement.

Selon A. Goodman, l'addiction est un regroupement de conduites définies par une dépendance compulsive, c'est à dire une conduite prioritaire, immaîtrisable, nocive et inflexible ${ }^{14}$ de la personne vis-à-vis d'un comportement (jeu d'argent, sexualité, alimentation, etc.) ou d'un produit psycho-actif(drogues). Retenons ici que le signe fondamental et commun à tous les courants de pensées est « la perte de liberté de s'abstenir $»^{15}$ de réaliser le comportement.

Cette définition uniquement descriptive de l'addiction laisse libre champ aux interprétations les plus variées concernant sa nature, ses causes et son traitement. De plus, la conception galénique de la psychiatrie moderne impacte plus sensiblement l'addiction en redoublant l'imaginaire « exogénétique » de la drogue. Elle permet la réinstauration de l'idée d'un sujet vidé de sa substance qui se trouverait possédé et dominé par l'objet démoniaque à savoir la drogue, le sexe ou le jeu. En ce sens, il y a rapidement confusion entre le trouble et l'objet d'addiction, l'objet honni serait à éliminer et la personne à renforcer, pour retrouver un sujet sain, délivré d'un comportement qui est préjudiciable d'une part pour sa liberté ${ }^{16}$ et d'autre part pour la société elle-même en termes de perpétuation et de participation au sens large ${ }^{17}$.

Dans le traitement médiatique et social de l'addiction s'expose la problématique au cœur même de l'addiction à savoir le couple responsabilité/ déresponsabilisation. Du point de vue psychique, le sujet se sentant responsable de son insuffisance trouve dans l'abandon de soi à l'objet d'addiction 
une déresponsabilisation vis-à-vis de ses idéaux interne et externe reportée sur l'illusion éphémère d'une maîtrise totale de l'objet. Au niveau social et médiatique, le sujet « addict » est responsabilisé en tant qu'il est bien le porteur du trouble, mais il en est simultanément dessaisi par la supposée toute-puissance de l'objet d'addiction. D'où les demandes cliniques et l'offre illusoire de certaines thérapeutiques d'ôter purement et simplement l'objet d'addiction comme si le sujet et la société n'étaient pas parties prenantes et qu'il n'y avait dans cet accrochage, aucune signification, aucune fonction. La dynamique propre à l'addiction trouve son reflet dans le traitement médiatique de celle-ci : l'objet masque le sujet.

Dès 1996, les tentatives d'application du modèle de l'addiction ${ }^{18}$ à l'usage problématique du jeu en ligne se basent donc sur une similarité de certains signes cliniques ou symptômes tels que la centration (monopole du jeu dans la vie du sujet), le retrait et l'appauvrissement (désinvestissement de la réalité sociale physique), la préoccupation (pensées autour de l'objet), la tolérance (besoin d'augmenter la quantité) et l'inflexibilité (persistance malgré la conscience des difficultés). Des signes importants de l'addiction tels que le manque, le craving ou compulsion, et l'impuissance (échec des tentatives d'arrêt) sont encore discutés repoussant l'intégration d'une entrée « addiction au jeu vidéo » dans le futur DSM. Officiellement, il n'y a donc pas d'addiction au jeu vidéo.

En France, aucune politique publique de santé ne subventionne de soins spécialisés, ni même de recherche sur le sujet, mais dans le même temps elle n'interdit pas à l'addictologie, spécialité psychiatrique chargée des addictions, d'offrir une prise en charge et des formations sur une maladie non reconnue. La captation des usages problématiques du jeu en ligne par l'addictologie amène ainsi au fait accompli : s'il existe une offre de soins spécialisée, il y a donc bel et bien une maladie relevant de cette spécialité. Institutionnellement l'addictologie constitue la vitrine publique des usages dépendants et toute nouvelle addiction tombera forcément dans son escarcelle comme la dépendance affective, le jeu vidéo et certainement d'autres en gestation, agrandissant par là même leur clientèle et sa place dans la communauté psychiatrique. Les conditions de naissance de la cyberdépendance auraient déjà dues attirées l'attention sur la motivation commerciale et médiatique d'une telle appropriation. Kimberly Young communiqua dans les milieux psychiatriques ${ }^{19}$ et créa une offre de soins payante dans la foulée de son étude pionnière et scientifiquement très discutable.

Les risques de l'usage du terme d'addiction appliqué au jeu vidéo en ligne sont donc :

1/ La stigmatisation d'une nouvelle pratique ludique, technologique et sociale encore en voie d'intégration et de régulation dans la population.

2/ La pathologisation et la surévaluation de pratiques excessives du MMORPG sur la base d'une description ignorant tant les dynamiques intrinsèques de l'objet que des différentes fonctions notamment antidépressives et compensatoires prises par le jeu pour un sujet donné.

3/ La captation centripète par des marchands de soins spécialisés au détriment d'une démarche d'explication psychosociale et anthropologique 
des mutations à l'œuvre dans la société et particulièrement dans le processus de subjectivation ${ }^{20}$.

\section{Le jeu vidéo : un objet curatif}

Les jeux vidéo représentent à l'heure actuelle une culture émergeante. Encore décriés comme un loisir source de violences et d'addictions, la réalité nous montre qu'ils représentent en terme de chiffre d'affaires, le premier des loisirs devant le cinéma, la vidéo et la musique depuis plus de trois ans. Cela veut dire que la plupart des enfants que nous recevons dans le cadre d'une psychothérapie d'inspiration psychanalytique rapportent de plus en plus souvent des histoires qui prennent la forme d'images (télévision, cinéma) et de plaisirs interactifs procurés par les jeux vidéo. Face à cet appauvrissement de l'imaginaire pour certains, il ne s'agit pas non plus de rejeter cette nouvelle approche du discours de l'enfant. En plus des images animées, qui sonnent comme des récits aussi riches que certains récits de rêves, je choisis le parti d'accorder autant de valeur tout en m'interrogeant sur ce nouveau type d'imaginaire qui passe plus par des représentations de choses que par des représentations de mots. Ces enfants ne veulent pas être « sages comme des images», et ils ne sont pas non plus des patients idéaux qui élaborent comme nous le souhaiterions. Les mots ne semblent pas avoir d'effets de mise à distance et les sentiments d'incomplétudes dans lequel ils vivent nous rappellent les blessures narcissiques qu'ils semblent avoir subies. Ces enfants croient à ce qu'ils voient et non plus à ce qu'ils pensent. Enfants païens, ils surinvestissent la pensée magique, souvent héritée de cultures traditionnelles, et vous expliquent que les fantômes existent bel et bien car ils les ont vus sur TF1!

\section{Penser en image et narcissisme}

Beaucoup d'enfants nés avec la télévision se sont souvent retrouvés dans des situations traumatiques où la télévision semblait être la seule image qui permettait de ne pas sombrer dans un effondrement dépressif. Elle fait du «bruit»et tient en éveil le psychisme sidéré ou au contraire sombrant dans les abîmes de l'image blanche de la dépression. Les mères «mortes» et/ou narcissiques, trouvent ce qu'elles peuvent pour aller mieux. Toxiques, maltraitance, le salon est illuminé par l'image de la télévision qui ranime, qui donne à voir des belles choses dans lesquelles on peut se projeter. L'enfant assis sur les genoux maternels, ou bien assis dans son fauteuil, face à la télévision, suit désespérément le fil du regard maternel. La mère regarde l'image de la télévision qui à l'inverse de son enfant ne sera pas vécue comme persécutrice ou bien la renvoyant à sa propre relation maternelle, souvent faite de relations pleines de tumultes et de haines. Ce tableau sombre reste pourtant un élément constituant une ébauche de troubles narcissiques diagnostiqués pour la plupart des enfants que nous recevons au C.M.P de Pantin. Ces bébés, amoureux du regard maternel, vont ainsi se tourner vers ce qui rend sa maman aussi captive. La mère captivée par la télévision, le bébé captivé par le regard maternel, la triangulation apparaît, mais d'une nature incomplète. La télévision ne nous parle ni ne nous répond. Mais cette expérience possède une valeur, celle d'une ébauche de médiatisation face à une relation duelle mortifère. De plus, 
elle témoigne d'une attention conjointe qui signe une évolution dans la relation symbiotique à la mère. Le miroir remplacé par la télévision, un début de symbolisation nécessaire se met en œuvre. Cette vignette clinique est un exemple de la force active du bébé pour trianguler à tout prix, pour ne pas être envahi par trop de pulsions de mort. La «mère morte » ne laisse pas beaucoup de choix et les images télévisées lui ont permis de ne pas sombrer dans le vide d'une non-relation. L'intrication pulsionnelle, passant par la valorisation narcissique fera de ce bébé un accroc des images. Ces images remplies d'illusion ont un sens, celles d'un paradis perdu, où le sourire maternel redonne de l'épaisseur à cette image perdue. Ces images feront ainsi l'effet d'une madeleine et à l'adolescence, pris dans ces mouvements mêlés de régression et de défi à l'autorité, regardera «Alien» comme une manière active et psychisée de lutter contre le mutant qui est en lui. Ces mouvements identificatoires, tout le monde les éprouvent. Mais pour «l'accroc», elles touchent à des identifications primaires qui feront que ces images l'envelopperont mais en même temps procureront des transformations dans ses désirs.

Les images n'auront pas uniquement le statut de loisir mais de messages envoyés à son inconscient dans un non-temps, propre à l'inconscient. Le «toxico» des images sera d'autant plus sensible à la forme de ces images, mais le fond est aussi important. Il ne pourra se satisfaire de tel type d'émission, car il ne s'agit que d'images en mouvement. La forme permet de donner du fond. Mais on peut s'interroger sur le fait que la forme vient surtout toucher des affects inconscients. Le fond ou l'histoire a plutôt affaire avec le pré-conscient.

Nous vivons dans une société où les représentations en images deviennent des repères incontournables. Ces re-pères n'en sont pourtant pas tout à fait. Il ne s'agit pas d'images qui tels les commandements divins sont d'ordre surmoïque mais plutôt d'exigences idéatoires. Notre société faites d'images propose des images qui donnent à voir ce que l'on peut avoir.

Si l'image devait être une forme de tiers paternel ou maternel, elle n'aurait pas la valeur de surmoi mais plutôt celle d'un idéal du moi tyrannique qui exige de nous d'être à son image. Les parents, eux-même pris dans ce engrenage sociétal véhiculent ces mêmes valeurs et les hissent en étendards de valeurs d'un bien être à tout prix. Notre société se « narcissise » et les images en sont les représentants. Pathologie de la représentation de soi, blessures narcissiques, pères défaillants, mère déprimée et/ou narcissique, les ingrédients sont présents pour engendrer des symptômes visibles ; à l'image des comportementaux qui n'envisage le patient que par ce qui est observable. Enfants hyperactifs, troubles du comportement violents, enfants carencés, ce sont ces enfants que nous recevons au CMP de Pantin.

\section{Le lien interactif et l'espace de projection}

Depuis maintenant quatre ans, je réfléchis d'un point de vue théorique sur ce nouveau lien : l'interactivité. En France, nous sommes encore trop peu de cliniciens à nous intéresser au jeu vidéo, envisagé comme une nouvelle forme de médiation thérapeutique. La plupart des soignants que j'ai rencontrés utilisant le jeu vidéo, s'avèrent 
être eux-mêmes des joueurs occasionnels. C'est d'ailleurs mon cas et en absence de cas clinique, je me suis pris au jeu de m'interroger sur mes mécanismes inconscients à l'œuvre dans le fait de jouer aux jeux vidéo.

J'ai pu donc depuis élaboré un début d'hypothèse clinique d'orientation psychanalytique, étayé depuis par des cas «d'accrocs au jeu vidéo » reçus en cabinet libéral, sur la pratique des jeux vidéo.

Les jeux vidéos, à l'inverse de ce que véhiculent la plupart des pouvoirs politiques et médiatiques, ne sont pas violents, dans le sens de Bergeret ${ }^{21}$, mais mettent en scène de l'agressivité, car contextualisée : " Je détruis les extra-terrestres car ils mettent en danger la planète ». Ce qui nous intéresse c'est de remarquer que la plupart des joueurs, dans des moments que l'on pourrait qualifier de baisse des pulsions de vie et d'émergence d'affects à résonance négative, vont pratiquer les jeux vidéo avec une intensité temporelle, parfois excessive. On pourrait évoquer le nombre de plus de deux heures de jeux quotidien. Ainsi, le jeu vidéo aussi bien dans sa relation au corps (Adolescence), par la pulsion d'emprise, que dans le contexte combatif, va permettre au Moi du sujet de se mettre en « colère ». Le jeu vidéo va fonctionner comme un antidépresseur virtuel par l'émergence de pulsions sadiques anales, tout en étant porté par une narration, à savoir l'histoire proposée par le jeu vidéo. Face à l'effondrement, le sadisme et/ou le masochisme reste du côté des pulsions gardiennes de la vie.

De plus le jeu vidéo, de par l'incarnation de l'avatar (double virtuel visible à l'image), per- met au joueur de ne pas incarner n'importe qui. Il s'agit en général de figures héroïques qui, dans un sentiment d'élection narcissiques, par des processus d'identifications primaires fait du joueur le metteur en scène et en même temps le spectateur du spectacle qu'il met en scène. $\mathrm{Ce}$ dispositif répare quelque chose des blessures narcissiques qui se ré-ouvrent à l'occasion de moments de fragilité où les autres renvoient le sujet à ses sentiments d'incomplétude. Pour exemple, un de mes patients en libéral, professeur agrégé à la Sorbonne, m'évoquait qu'après certaines humiliations que lui faisaient subir sa femme, il se précipitait sur le jeu vidéo, « Sim’s City ", où dans un phénomène que nous pourrions nommer de «main régnante », mettait en scène ses fantasmes grandioses, où l'inhibition n'a pas de place.

Mais ce qui est sûrement le plus fascinant dans les jeux vidéo est cet autre virtuel que l'on nomme, à tort, l'intelligence artificielle. Il s'agit en effet d'une illusion d'intelligence artificielle, qui fait que lorsque vous jouez seul, les ennemis ont été programmés pour vous empêcher d'être dans le «tout, tout de suite». Ces personnages programmés, qui sont en fait la plupart du temps, des ennemis, ont pour vocation de nous « mettre des bâtons dans les roues ». Figures rivales, elles représentent le tiers indispensable entre le joueur et le jeu vidéo.

Encore une fois, à l'inverse de ce que pensent beaucoup de détracteurs des jeux vidéo, ils ne font rarement émerger que de la décharge. $\mathrm{Ce}$ qui est donc intéressant d'un point de vue de l'utilisation thérapeutique c'est que ces figures amicales ou rivales, créées par le programmeur $\mathrm{du}$ 
jeu, va servir au joueur de surface de projection de ses propres figures. Ces figures prennent souvent l'allure d'imagos parentaux et vont, comme dans finalement toute œuvre d'art, faire émerger des processus inconscients d'identification et de contre-identification.

Pour ces enfants qui " pensent en image », le jeu vidéo pourra même de manière solitaire avoir une vertu auto-thérapeutique. Le danger se situera dans l'aspect addictif où l'objet jeu vidéo ne sera pris que comme une « machine à tuer le temps », ou plus précisément il ne sera pas accompagné de représentations verbales.

Penser en images implique que les représentations et affects soient liés par une dimension qui devient visible. L'hallucination du Sein est le premier acte de penser chez l'être humain, et pourtant, il y a comme un défaut d'intégration de cette capacité à se représenter les choses en son absence, pour la plupart des enfants, adolescents et jeunes adultes que nous recevons. Nous ne sommes pas dans des pathologies psychotiques, et l'accès au symbolique semble se faire plutôt du côté de l'Idéal du Moi que du Surmoi. Devant cet état de fait, l'utilisation des images comme moyen de faire ré-émerger le langage semble être la voie à choisir. Images violentes ou violence d'un monde fait d'images, le danger est d'accorder trop d'importance à ces dernières. L'être humain reste l'auteur, mais c'est au spectateur d'être vigilant s'il ne veut pas prendre des images pour des états de fait. C'est sûrement dans le lien interactif et plus précisément dans le jeu vidéo que pourrait se situer une voie intéressante pour métaphoriser, transformer cet idéal pour en faire une histoire dans laquelle on pourrait interagir.

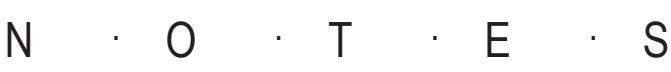

1. Winnicot, Jeu et réalité, 1975, Folio essais, 2002

2. T. Gaon, « Des mondes numériques comme paradis artificiels" in Culture d'univers, F. Beau (dir.), Limoges, FYP, 2007, pp. 116-129.

3. Rapidement rebaptisés Evercrack et NeverRest pour son aspect addictif, Everquest est le boutefeu de l'addiction au jeu vidéo aux USA avec le suicide du joueur Shawn Woolley, la médiatisation et la judiciarisation de l'affaire par sa mère qui créera par la suite le premier groupe néphaliste sur ce problème On-Line Gamers Anonymous (joueurs en ligne anonyme).

4. N. Yee, «The Daedalus Project». Disponible sur Internet : http://www.nickyee.com/daedalus. S.M. Grüsser, R. Thalemann et M.D. Griffiths (2007), «Excessive computer game playing : Evidence for addiction and aggression? » In Cyberpsychology \& Behavior, $1^{\circ}$, 2, pp. 290-292.

5. Voir les conflits d'intérêts entre l'industrie pharmaceutique et les experts de la psychiatrie in Lisa Cosgrovea, Sheldon Krimsky, Manisha Vijayaraghavana, Lisa Schneidera, Financial Ties between DSM-IV Panel Members and the Pharmaceutical Industry, University of Massachusetts, Boston, Mass., and Tufts University, Medford, Mass. Psychotherapy and Psychosomatics, 2006 ; 75 : 154-160 (vol. 3, avril 2006).

6. Voir à ce sujet A. Ehrenberg, La fatigue d'être soi, Paris, Odile Jacob, 2000, pp. 194-195.

7. A. Rigaud et M. Maquet « Propos critiques sur les notions d'addiction et de conduites de dépendance. Entre lieux communs et chimères ", in D. Bailly et J-L. Venisse (dir.) Dépendances et conduites de dépendance, Paris, Masson,1994.

8. La sémiologie d'une maladie est l'ensemble des 
signes cliniques permettant de l'identifier.

9. Pour Galien la maladie est étrangère au corps, elle est abordée de façon analytique en isolant les symptômes pour leur attribuer des causes distinctes. Pour Hippocrate la maladie est inhérente à la condition humaine, elle est abordée de façon globale prenant en compte les symptômes et la personnalité, l'histoire et l'environnement du patient.

10. En 1975, Stanton Peele établit un parallèle entre la dépendance aux drogues et la dépendance (psychique) à une personne dans le cadre d'un couple dans son ouvrage Love and addiction, New York, Taplinger.

11. L'utilisation du champ lexical de l'addiction pour décrire l'envie ou le besoin du produit en rend compte, tel le parfum « Dior Addict» ou le slogan de Sega « C'est plus fort que toi ».

12. Appelées nouvelles addictions comportementales. Elles comprennent communément: le jeu pathologique ou gambling, la sexualité compulsive, les achats compulsifs, la boulimie.

13. Le terme est introduit par J. McDougall, Plaidoyer pour une certaine anormalité, Paris Gallimard,1978, pp.198-199. J. Bergeret le développera sur le registre de la dette à payer par son corps dans Le psychanalyste à l'écoute du toxicomane, Paris, Dunod, 1981.

14. Persévérant malgré la conscience des difficultés. 15. Aphorisme de P. Fouquet à propose de la dépendance, 1951. La dépendance c'est donc ne pas pouvoir s'arrêter malgré la volonté.

16. C'est en tant que pathologie de la liberté que la dépendance entre dans le champ de la psychiatrie, car selon H. EY « Le champ de la psychiatrie (est) celui de la pathologie de la liberté » in H. EY, Des Idées de Jackson à un modèle organo-dynamique en Psychiatrie, Privat, 1975.

17. Sur la conception française de l'addict comme un être antisocial ayant perdu son statut primordial de citoyen, voir A. Ehrenberg, L'individu incertain,
Paris, Calmann Levy, 1995, pp. 80-91.

18. Dans l'étude de KS. Young, le jeu en ligne est une sous-catégorie de la cyberdépendance dont le modèle et les critères sont issus du jeu pathologique, addiction reconnu par le DSM-III en 1980. Young KS. « Internet addiction: the emergence of a new clinical disorder » in Cyberpsychol Behav. 1996 ;1:237-244.

19. K. Young, «What makes the Internet addictive? Potential explanations for pathological Internet use », présenté au $105^{\mathrm{e}}$ Congrès annuel de 1'Association Américaine de Psychiatrie (A.P.A.), Chicago, 1996. 20. Voir à ce sujet A. Ehrenberg, L'individu incertain, Calmann Levy, Paris, 1995.

21. J. Bergeret, La Violence Fondamentale, Dunod, 2003. 
$R \cdot E ́ \cdot S \cdot U \cdot M \cdot E ́$

La problématique de l'addiction aux jeux vidéo pose la question de leurs effets performatifs. La notion d'addiction, ici fortement critiquée, se révèle être avant tout un enjeu doctrinal pour la psychologie clinique. Cette généalogie détaillée du concept remet en cause la notion même d'addiction et privilégie un usage excessif d'un genre particulier de jeux, les jeux de rôle massivement multi-joueurs. Ces deux regards croisés sur les jeux vidéo révèlent ainsi la dimension transitionnelle de ces jeux vidéo dès lors qu'ils sont investis par le joueur. L'usage thérapeutique du jeu vidéo, offre un contre-point intéressant sur la capacité de ces jeux à permettre l'expression d'angoisses chez les plus jeunes. Au final, cette perspective psychanalytique remet en cause le déterminisme technologique en privilégiant la pluralité des usages.

\section{Healing from / with videogames}

The problem of addiction to videogames raises the question of their performing effects. Addiction concept, strongly criticized here, appears to be primarily a doctrinal issue for clinical psychology. A detailed genealogy of this notion calls into question the very concept of addiction and focuses on an excessive use of a particular gender of games : massively multiplayer role-playing games (MMORPG). These two crossed visions on videogames reveal the transitional dimension of videogames as soon as they are invested by player. Therapeutic use of video game offers an interesting counter-point on these games ability to allow the expression of anguish with young patients. In the end, this psychoanalytic perspective limits the technological determinism by focusing on multiple uses. 